\title{
Framework based on Gestalt principles to design mobile interfaces for a better user experience
}

\author{
Daniel Ripalda ${ }^{1}$, César Guevara ${ }^{1}$ and Alejandra Garrido ${ }^{2}$ \\ ${ }^{1}$ Indoamérica University, Mechatronics and Interactive Systems Research Center \\ Machala \& Sabanilla, Quito, Ecuador \\ ${ }^{2}$ LIFIA, Fac. de Informática, Univ. Nac. de La Plata \& CONICET \\ $50 \& 120,1900$ La Plata, Argentina \\ 1danielripalda@uti.edu.ec 22garrido@lifia.info.unlp.edu.ar
}

\begin{abstract}
This paper presents the results of the user experience test comparing a real functional application and a high fidelity prototype that used a Framework to design graphic user interfaces on mobile devices. This Framework links Nielsen's heuristics with the principles of perception of Gestalt, offering to developers and usability experts, references to generate and evaluate mockups and prototypes. The constructive and evaluative model of the Framework allows to recognize usability criteria in visual components of the interfaces, during the initial phases of a project that uses agile software development methodologies, reducing the "trial - error" regressions. The experiment allowed obtaining data about satisfaction measures and specific user attitudes regarding the interfaces developed.
\end{abstract}

Keywords: Gestalt, User Interfaces, Usability, Mobile, Agile Software Development.

\section{Introduction}

There are different reasons why mobile applications fail, one of the most recurring factors is that the Graphical User Interface (GUI), produces unfavorable results to the user experience [1], the development team constantly seeks to balance aspects of usability with visual aesthetics while achieving efficiency, effectiveness, and satisfaction [2]. The problem of designing interfaces to obtain satisfactory usability has been partially solved using models, concepts and processes such as Nielsen's Heuristics [3], User-Centered Design [4], Pattern-Based Design [5] or Mockups [6]. In the specific case of the heuristics, different schemes describe it in a visual way, but those references are generic, non-concrete and insufficient to execute a project, mainly because they open a wide range of possible interpretations.

The usability experts of a team could believe that they are fulfilling Nielsen's heuristics while the prototype tests continually show errors, which the group fails to detect because they think that the interfaces are correct. Regressions after negative results in user experience tests can delay a project significantly; in fact, agile development methodologies contemplate these regressive cycles [7] [8] as normal. With the goal of improving the support given by heuristics to the usability experts in 
the early phases of a development project, a previous work presented the theoretical approach of the Gestalt Prototyping Framework [9] that relates Nielsen's heuristics with Gestalt's principles, this work extends to a first experimental trial of the Framework simulating a development stage for a real application.

\section{Related work}

In Computer Science as in other the fields of knowledge, there are universally recognized principles like Morgan's Law and Heuristics. The search of universal paradigms has also occurred in other disciplines of human sciences as Psychology, Arts, Design and Architecture. An example of this kind of universal foundation are the Gestalt principles, which explain the way in which humans perceive and recognize visual patterns. Graphical user interfaces allow interaction and facilitate the perception of the human being [10], therefore the Laws of the Gestalt naturally are already in the screen of a device, because the components of an interface are related and interact with each other all the time, influencing the level of development of the tasks that a user executes in a mobile application. This knowledge has been used in different computer systems [11], in order to understand how a user generates the actions she performs in an interface [12] [13] [14] [15]. However, previous work has not investigated in-depth how to use Gestalt principles in the design of interactions on mobile devices.

\section{Gestalt Prototyping Framework}

In the Development of interfaces, the Gestalt principles define the interaction of the graphic components, while the Heuristics move as guides to design the humancomputer interaction. Normally, both concepts are considered separately.

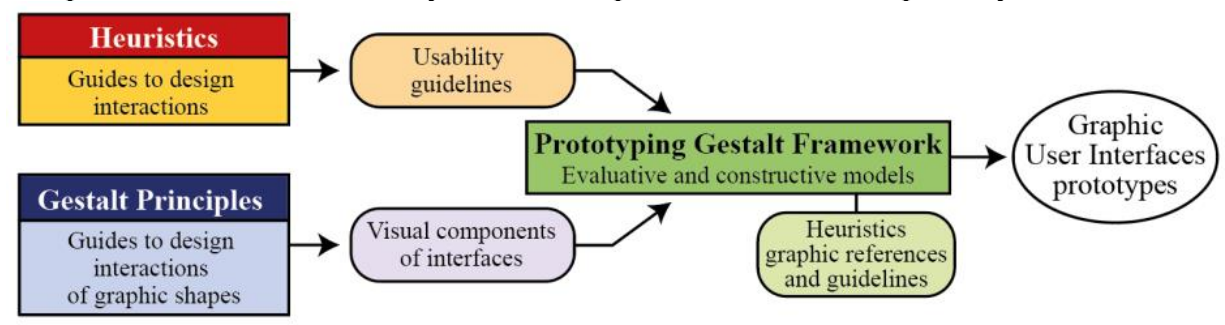

Fig. 1. Gestalt Prototyping Framework.

The Framework has a constructive model, which guides the development team during the production of high and low fidelity interfaces, describing components and specific interaction. In the definition of the Framework, we have linked Nielsen Heuristics to Gestalt principles, based on an analysis of the similarities in the statements and the graphic representations available in different sources, according to Table 1. Additionally, we have developed an evaluation model that allows usability experts to verify the components of a GUI before and after a user experience test. The following subsection describe 3 heuristics and the associated Gestalt principles. 
Table 1. The link between Nielsen's Heuristics and Gestalt principles.

\section{Nielsen's Heuristics}

Match between system and the real world

Recognition rather than recall

Aesthetic and minimalistic design
Gestalt principle

Similarity

Proximity - Common direction

Simplicity

\subsection{Statement 1 . The match between the system and the real world.}

The visual components [16] should be used to facilitate the user to recognize and associate each one with the activities that she may perform. Table 2 describe the Heuristic, the Gestalt principle and the components involved in one interactions.

Table 2. Gestalt Prototyping Framework Statement 1, Constructive Model.

\begin{tabular}{|l|l|}
\hline \multicolumn{1}{|c|}{ Nielsen's heuristic } & \multicolumn{1}{c|}{ Gestalt principle } \\
\hline Match between system and Real World: & Similarity: \\
The system should speak the users' language, & $\begin{array}{l}\text { If an element has similar characteristics to } \\
\text { making information appear in a natural and } \\
\text { others, it will be perceived as part of a group } \\
\text { regardless of distance }\end{array}$ \\
\hline logical order. & Usability interactions: \\
Interface Components: & Learnability, and Efficiency \\
\hline
\end{tabular}

In Figure 2, (a) there are two groups of icons with the same visual concept and color but with a different layout; in case of (b), the random placement is notorious and confusing, which evidencies the need for a reclassification task. This principle has been used in games like Candy Crush (c).

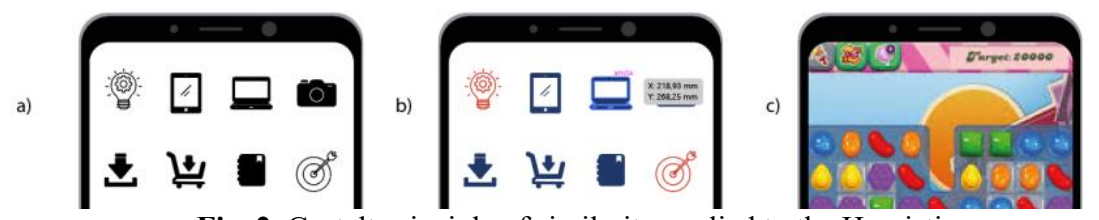

Fig. 2. Gestalt principle of similarity applied to the Heuristic.

\subsection{Statement 2. Recognition rather than recall}

To reduce the cognitive load during the phase of acquisition of skills, the menus and the relevant information of the application must appear, disappear and move in the interface, grouped by importance and function. Table 3 shows...

Table 3. Gestalt Prototyping Framework Statement 2, Constructive Model

\begin{tabular}{|l|l|}
\hline \multicolumn{1}{|c|}{ Nielsen's heuristic } & \multicolumn{1}{c|}{ Gestalt principles } \\
\hline Recognition rather than recall: Minimize & Proximity: \\
the user's memory load by making objects, & Nearby elements are perceived as a unit. \\
actions, and options visible. The user should & Common direction: \\
not have to remember information from one & $\begin{array}{l}\text { Elements moving in the same direction are } \\
\text { part to another. }\end{array}$ \\
\hline
\end{tabular}




\begin{tabular}{|l|lr|}
\hline $\begin{array}{l}\text { Interface Components: Menus - submenus, } \\
\text { Pop-up information, Fixed, and sliding } \\
\text { buttons. }\end{array}$ & $\begin{array}{l}\text { Usability interactions: Learnability, } \\
\text { Efficiency, and Memorability }\end{array}$ \\
\hline
\end{tabular}

Figure 3 shows that grouping similar buttons are perceived as menus (a), the menus can change position to reconfigure the interface (b), the elements of a mobile interface that slide towards the same position are also perceived as a group with the same function, this can be visualized in the user histories in the interface of Instagram (c).
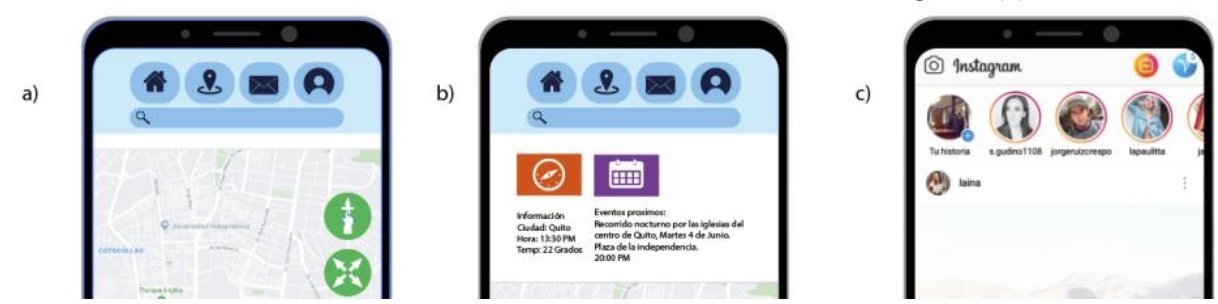

Fig. 3. Gestalt principles of Proximity and common direction applied to the Recognition Heuristic

\subsection{Statement 3, Aesthetic and minimalistic design}

For a mobile application to be easy to use, it has to reduce the visual information of an interface, eliminating obvious elements so that the relevant thing can stand out.

Table 4. Gestalt Framework Statement 2, Constructive Model

\begin{tabular}{|l|l|}
\hline \multicolumn{1}{|c|}{ Nielsen's heuristic } & \multicolumn{1}{c|}{ Gestalt principles } \\
\hline Aesthetic and minimalistic design & Simplicity \\
Interfaces should not contain information & Human beings organize perceptual fields \\
which is irrelevant or rarely needed. & with simple and regular features. \\
\hline Interface Components & Usability interactions \\
Backgrounds and Divs & Memorability and Usefulness \\
\hline
\end{tabular}

The Gestalt principle indicates that the figures can be perceived complete even if only a portion of them are shown; the funds and the distribution of the interface must reduce unnecessary elements. Figure 4 shows an interface with an excess of graphic details (a), the GUI can maintain the same elements reducing the visual information without affecting the functionality (b), the most successful apps use simple graphic (c).
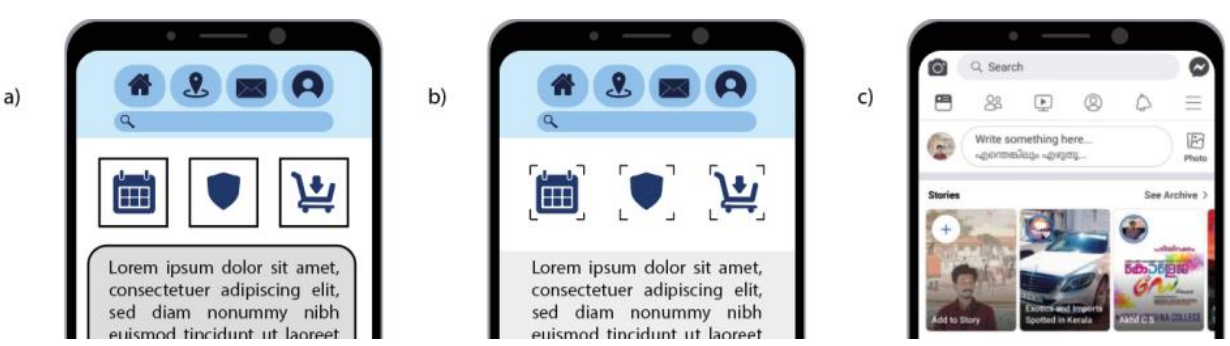

Fig. 4. Gestalt principles of Proximity and common direction applied to the Heuristic 


\section{Experiment and Results}

Using concepts related to the Gestalt Prototyping Framework, we made an experiment to rate 10 of the most recognized applications of the Android platform [17]. Table 5 shows the results of the parameters learnability (a), user satisfaction (b), ease of use (c), usefulness (d), measured using a Likert $(1-5)$ scale range, together with Gestalt principles identified.

Table 5. App rate using the Gestalt Prototyping Framework results

\begin{tabular}{lcccccl}
\hline & a & b & c & d & Value & Gestalt principles found \\
\hline Facebook & 5 & 5 & 4 & 5 & 19 & Symmetry, continuity, simplicity, closure \\
\hline Twitter & 3 & 4 & 5 & 4 & 16 & Symmetry, continuity, simplicity, closure \\
\hline Youtube & 4 & 4 & 4 & 4 & 16 & Symmetry, continuity, simplicity, proximity \\
\hline Uber & 3 & 5 & 3 & 4 & 15 & Symmetry, continuity, simplicity, proximity \\
\hline Hi5 & 4 & 4 & 4 & 3 & 15 & Symmetry, closure \\
\hline Vibbdi & 4 & 3 & 3 & 4 & 14 & Experience, symmetry, closure \\
\hline Vimeo & 4 & 3 & 3 & 4 & 14 & Experience, symmetry, closure \\
\hline One Search & 3 & 3 & 4 & 4 & 14 & Experience, simplicity \\
\hline InDrive & 3 & 3 & 3 & 3 & 12 & Experience \\
\hline Wikipedia & 2 & 3 & 2 & 3 & 10 & Not found
\end{tabular}

The App with the least index value was Wikipedia updated version in September 2019, using the Framework graphic references, the development team created low and highfidelity offline prototypes. The proposed changes in the interface did not focus on aesthetic aspects or replacement of the main functions. To obtain comparative results, a user experience test was developed on the real application and the high fidelity prototype developed in Adobe XD and executed on the Android platform. Figure 5 shows the original App (a) and the screens of the prototype in Adobe XD (b).

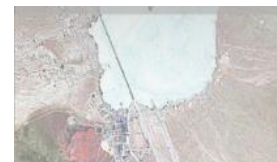

Área 51

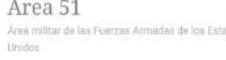

EA Área 51, también conocida como Groom Lake o Homey Aitport (OACl: KCTA), es un

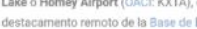

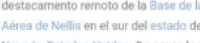
Cin lan designaciones correctus para instal Alimacenado en ista de guartiades

a)

$\boldsymbol{n}<\bar{x}_{A} \pi$

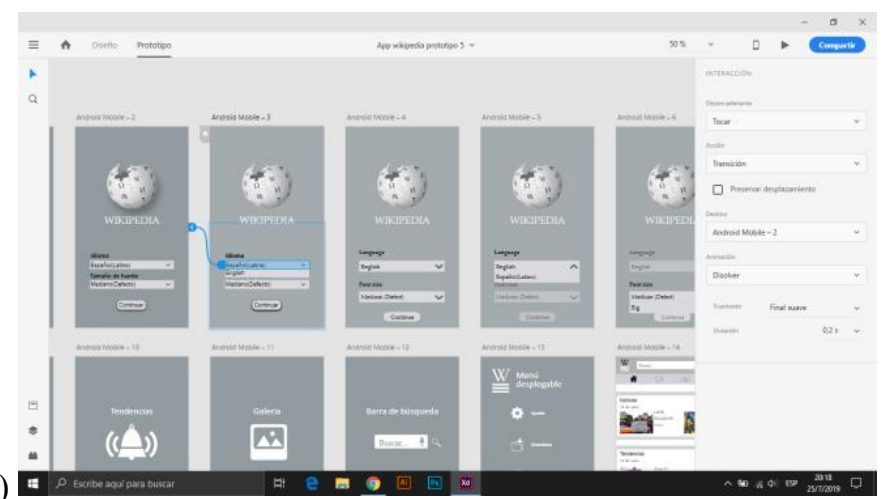

Fig. 5. Desarrollo del prototipo para las pruebas de usuario 
The experiment worked with one randomized group of 12 people, including teachers and students of the Indoamérica University, whose age range was between 20 and 45 years. The used instrument, employed 32 interaction screes and 8 tasks developed in defined periods of time. After each task the participant noted their own user experience data using Likert scale ranges ( [18] [19] ); the experiment was recorded in a video closed circuit and during the test, the screens were captured.

The activities performed by the users during the test were: Task1: "Run the application and go to the home screen"; Task 2: "From the home page go to the trends section"; Task 3: "Find a trending topic and share it with an external application"; Task 4: "Go to the news section, find the topic Area 51"; Task 5: "Open the image preferences in the topic and return to the home screen", Task 6. "Find the trending topic Greece and save it as a favorite". Task 7. "Go to the home page and then find the page you saved", Task 8. "Open the preferences of the App and change the language". The next table presents the average results of user experience test, comparing the real mobile application with the high fidelity prototype. The parameters measured were speed (a), ease of use (b) and preferred interface parameters (experience, learnability, ease).

Table 6. Experiment results

\begin{tabular}{|c|c|c|c|c|c|c|c|c|c|}
\hline & \multicolumn{3}{|c|}{ Real Application } & \multicolumn{3}{|c|}{ Prototype } & \multicolumn{3}{|c|}{ Preferred interface } \\
\hline & $\mathrm{a}$ & $\mathrm{b}$ & rate & $\mathrm{a}$ & $\mathrm{b}$ & rate & Experience & Learnability & Ease \\
\hline Task 1 & 4 & 4 & 8 & 4 & 5 & 9 & \multirow{4}{*}{$\begin{array}{c}\text { Prototype } \\
90 \%\end{array}$} & \multirow{4}{*}{$\begin{array}{c}\text { Prototype } \\
95 \%\end{array}$} & \multirow{4}{*}{$\begin{array}{c}\text { Prototype } \\
100 \%\end{array}$} \\
\hline Task 2 & 2 & 3 & 5 & 3 & 4 & 7 & & & \\
\hline Task 3 & 3 & 1 & 4 & 4 & 4 & 8 & & & \\
\hline Task 4 & 3 & 3 & 6 & 4 & 5 & 9 & & & \\
\hline Task 5 & 3 & 2 & 5 & 4 & 4 & 8 & \multirow{4}{*}{$\begin{array}{l}\text { Real } \\
10 \%\end{array}$} & \multirow{4}{*}{$\begin{array}{c}\text { Real } \\
5 \%\end{array}$} & \multirow{4}{*}{$\begin{array}{l}\text { Real } \\
0 \%\end{array}$} \\
\hline Task 6 & 2 & 1 & 3 & 3 & 4 & 7 & & & \\
\hline Task 7 & 1 & 1 & 2 & 3 & 3 & 6 & & & \\
\hline Task 8 & 2 & 2 & 4 & 4 & 4 & 8 & & & \\
\hline & \multicolumn{2}{|c|}{ Total } & 37 & \multicolumn{2}{|c|}{ Total } & 62 & & & \\
\hline & \multicolumn{2}{|c|}{ Perform. } & $46,2 \%$ & \multicolumn{2}{|c|}{ Perform. } & $77,5 \%$ & & & \\
\hline
\end{tabular}

\section{Conclusions and future work}

Using the Gestalt Prototyping Framework, the development team was able to identify efficiently the different visual components of the interface in the mobile application of Wikipedia, which had a negative impact an affect the results of the user experience test of the actual application. Improving these specific elements based on the guidelines of the Gestalt, without the need to alter the operation of the application, it is possible that the users choose the proposed interface while at the same time there is an increasing perception of ease and speed by $31,3 \%$. In the immediate future, it is necessary to test the integration of the Framework into a completely new development project, preferably some that use agile methodologies, to determine if according to expectations, it can reduce the time in production and trial-error regressions. 


\section{References}

1. Garrido, A., Firmenich, S., Grigera, J., \& Rossi, G, «Data-Driven Usability Refactoring: Tools and Challenges,» de 2017 6th International Workshop on Software Mining (SoftwareMining), 2017.

2. Marcus, A., «The cult of cute: The challenge of user experience design interactions,» pp. 2934, 2002.

3. J. Nielsen, «Ten usability heuristics.,» 2005.

4. Abras, C., Maloney-Krichmar, D., «User-centered design,» de Encyclopedia of HumanComputer Interaction, Thousand Oaks: Sage Publications, (2004), pp. 445-456..

5. Granlund, Å., Lafrenière, D., « A pattern-supported approach to the user interface design process,» International Conference on Human-Computer Interaction, 2001.

5. Rivero, J. M., Grigera, J., Rossi, G., Luna, E. R., Montero, F., \& Gaedke, M., « Mockupdriven development: providing agile support for model-driven web engineering,» Information and Software Technology, 2014.

7. Schwaber, K. , «Scrum development process,» Business object design and implementation, pp. 117-134, 1997.

3. Letelier, P., \& Penadés, M. C., «Agile methodologies for software development: eXtreme Programming (XP),» 2006.

7. D. Ripalda, A. Garrido y C. Guevara, «Gestalt framework in the design of interfaces for mobile devices: Theoretical approach,» de 13th Iberian Conference on Information Systems and Technologies (CISTI), Cáceres, Spain, 2018.

10. Graham, L., «Gestalt theory in interactive media design,» Journal of Humanities \& Social Sciences, 2008.

11. J. Gómez, Measuring the Impact of Gestalt Theories on Graphic user interface design, 2011.

12. Xiang, P., Yang, X., \& Shi, Y., Web page segmentation based on gestalt theory, In Multimedia and Expo, 2007 IEEE International Conference on (pp. 2253-2256). IEEE., 2007.

13. Looije, R., te Brake, G. M., \& Neerincx, M. A., Usability engineering for mobile maps., Proceedings of the 4th international conference on mobile technology, applications, and systems and the 1st international symposium on Computer human interaction in mobile technology, 2007.

14. Gong, J., \& Tarasewich, P., Guidelines for handheld mobile device interface design, Proceedings of DSI 2004 Annual Meeting (pp. 3751-3756)., 2004.

15. King, M. T., Grunbock, C., Mannby, C. F., \& Valenti, W., U.S. Patent Application 12/961,407., 2010

16. J. Moyes y P. Jordan, Icon design and its effect on guessability, learnability, and experienced user performance., People and computer, 1993.

17. Kumar, B. A., \& Mohite, P. , «Usability of mobile learning applications: a systematic literature review,» Journal of Computers in Education, pp. 1-17, 2018.

18. Hornbæk, K., «Current practice in measuring usability: challenges to usability studies and,» Int. J. Hum Comput Stud, pp. 72-102, 2006.

19. Cajas, V., Urbieta, M., Rybarczyk, Y., Rossi, G., \& Guevara, C, «An Approach for Migrating Legacy Applications to Mobile Interfaces,» World Conference on Information Systems and Technologies, pp. 916-927, 2019. 